BULLETIN OF THE

AMERICAN MATHEMATICAL SOCIETY

Volume 80, Number 5, September 1974

\title{
A REPRESENTATION OF CLOSED, ORIENTABLE 3-MANIFOLDS AS 3-FOLD BRANCHED COVERINGS OF $S^{3}$
}

\author{
BY JOSÉ M. MONTESINOS
}

Communicated by William Browder, April 5, 1974

A represented link is a link $L$ in $S^{3}$ together with a representation $\omega$ of the link group $\pi\left(S^{3}-L\right)$ into the symmetric permutation group of $d$ symbols $\mathscr{S}_{d}$. Let us call $\omega$ simple if it represents each meridian of $L$ by an appropriate transposition. If $(L, \omega)$ is a represented link, there is a uniquely associated closed, orientable 3-manifold $M(L, \omega)$, namely the $d$-fold covering of $S^{3}$ branched over $L$, that is determined by the representation $\omega$. It has been proved by J. W. Alexander [1] that every closed orientable 3-manifold is $M(L, \omega)$ for some link $L$ and representation $\omega$.

H. M. Hilden (personal communication to the author) has proved

THEOREM 1. Every closed, orientable 3-manifold is $M(K, \omega)$ for some knot $K$ and simple representation $\omega$ of $\pi\left(S^{3}-K\right)$ onto $\mathscr{S}_{3}$.

Theorem 1 states that every closed, orientable 3-manifold is a 3-fold irregular covering space of $S^{3}$ branched over a knot $K$ in such a way that the inverse image of a point of $K$ consists of a point of branch-index 2 and a point of branch-index 1 .

We have obtained (independently) a different proof of Theorem 1 which will be sketched here. A detailed proof will appear elsewhere.

Let $L$ be a link in $S^{3}$ composed of two unlinked trivial knots $P$ and $R$, and let $\omega$ be a representation of $\pi\left(S^{3}-L\right)$ onto the group $\mathscr{S}_{3}$ of permutations of the symbols 0,1 , and 2 , such that $\omega$ represents a meridian of $P$ (resp. $R$ ) by the transposition (01) (resp. (02)). It is easy to see that $M(L, \omega)$ is $S^{3}$. Let $p: M(L, \omega) \rightarrow S^{3}$ be the covering projection. Then $p^{-1}(P)$ (resp. $p^{-1}(R)$ ) is composed of a curve $P_{01}$ (resp. $R_{02}$ ) of branchindex 2 and a curve $P_{2}$ (resp. $R_{1}$ ) of branch-index 1 . These curves are unknotted and unlinked. Let $B$ be a ball in $S^{3}$ which cuts $L$ in exactly two disjoint arcs in $P$ and such that $p^{-1}(B)$ is the disjoint sum of a ball $B_{2}$ which cuts $P_{2}$, and a solid torus $B_{01}$ which cuts $P_{01}$. Then $p \mid B_{01}: B_{01} \rightarrow B$ is

AMS (MOS) subject classifications (1970). Primary 55A10, 57A10; Secondary 55A25.

Key words and phrases. Branched covering space, surgery on link, three-manifold, knot, link.

Copyright (c) American Mathematical Society 1974 
the branched covering induced by the "symmetry with respect to axis $P_{01}$ ". If we remove $B$ from $S^{3}$ and resew them differently, in such a way that $\partial B \cap P$ keeps fixed as a set, the effect in the covering is to remove $p^{-1}(B)$ from $S^{3}$ and to resew it differently. This is the same as doing surgery on the solid torus $B_{01}$.

W. B. R. Lickorish has proved [2, p. 419] that every closed, orientable 3-manifold can be obtained by surgery on a member of a special family $\mathscr{F}$ of links in $S^{3}$. If $N^{\prime}$ is a link in $\mathscr{F}$, there is a link $N$, of the same isotopy type as $N^{\prime}$, such that each component of $N$ cuts $P_{01}+R_{02}$ in exactly two points and is "symmetric" with respect to $P_{01}$ or $R_{02}$. If $N$ has $n$ components, then there is a union of $n$ disjoint solid balls $B_{1}, \cdots, B_{n}$ such that $p^{-1}\left(\bigcup_{i=1}^{n} B_{i}\right)$ is the disjoint union of a regular neighbourhood of $N$ and $n$ solid balls in $M(L, \omega)$. We can remove the balls $B_{1}, \cdots, B_{n}$ from $S^{3}$ and resew them differently in order to obtain a link with a simple representation onto $\mathscr{S}_{3},\left(L^{\prime}, \omega^{\prime}\right)$, such that $M\left(L^{\prime}, \omega^{\prime}\right)$ is exhibited as a manifold obtained by doing a given surgery on the link $N$. This shows that each manifold obtained by surgery on a member of $\mathscr{F}$ is $M\left(L^{\prime}, \omega^{\prime}\right)$ for some link $L^{\prime}$ and some simple representation $\omega^{\prime}$ onto $\mathscr{S}_{3}$. Now, we can apply the modifications defined in [3] to $\left(L^{\prime}, \omega^{\prime}\right)$ to obtain a knot with a simple representation onto $\mathscr{S}_{3},\left(K, \omega^{\prime \prime}\right)$, such that $M\left(K, \omega^{\prime \prime}\right)=M\left(L^{\prime}, \omega^{\prime}\right)$. This proves Theorem 1.

Note that our proof is constructive in the following sense. If a manifold $M$ is obtained by surgery on a member of $\mathscr{F}$, we can exhibit a simple represented $\operatorname{knot}(K, \omega)$ such that $M(K, \omega)=M$.

\section{REFERENCES}

1. J. W. Alexander, Note on Riemann spaces, Bull. Amer. Math. Soc. 26 (1920), 370-372.

2. W. B. R. Lickorish, A foliation for 3-manifolds, Ann. of Math. (2) 82 (1965), 414-420. MR 32 \#6488.

3. J. M. Montesinos Amilibia, Reduction of the Poincaré conjecture to other geometric conjectures, Rev. Mat. Hisp.-Amer. (4) 32 (1972), 33-51. (Spanish) MR 47 \#2590.

Facultad de Ciencias, Sección de Matemáticas, Universidad Complutense, MADRID, SPAIN 